\title{
Analysis of heterogeneous information and diagnostics of complex technical systems based on methods of diakoptics and correlation analysis
}

\author{
Nuri D. Narakidze, Roman G. Oganyan*, and Vasily A. Mokhov \\ Platov South-Russian State Polytechnic University (NPI), 346400 Novocherkassk, Russia
}

\begin{abstract}
The article is devoted to the investigation of the applicability of the correlation analysis for the processing of heterogeneous data obtained from complex technical systems. In this article, heterogeneous data is understood to mean heterogeneous data obtained from means of monitoring the state of individual objects. The proposed approach is proposed to be used to diagnose complex technical systems using the example of digital substations. For this purpose, an imitation model for power substation based on the method of diakoptics was developed. And all components of the system, regardless of their physical functioning principles, are represented in the form of electrical sub-models. The substitution schemes based on operational amplifiers are used. The model simulates the operation of measuring current and voltage transformers, power transformer, circuit breaker and relay protection. To test the proposed approach, we also considered an amplifier circuit based on three operational amplifiers. The calculation of the circuit is described, as well as the simulation of the circuit in the MicroCap environment. The simulation was carried out with the aim of realizing parametric and structural diagnostics on the basis of correlation analysis. The results of modeling the applicability of the proposed approach for both parametric and structural diagnostics is proved operability.
\end{abstract}

\section{Introduction}

As known the innovative level of automation of traditional substations are digital substations, which are now being introduced everywhere [1-3]. A digital substation, like any complex technical system, involves a large number of elements and interactions between them. The most common approach to analyzing data from primary sensors in the diagnosis of such systems is to set the limits of the monitored parameters and then to compare with them (threshold analysis). Currently, threshold analysis is used to identify current and developing damage to individual components of the system [4]. It is also worth highlighting various methods of intellectual analysis, but in general they are also focused on a single element of the system $[5,6]$.

\footnotetext{
* Corresponding author: roman.work18@gmail.com
} 


\section{Materials and Methods}

An idea is proposed that consists in combining information (or data) from various sources (or from different sensors) in order to achieve better evaluation results (for example, accuracy and reliability) than from one source. A number of studies [7-12] showed that combining heterogeneous information about a diagnosed object allows obtaining more accurate diagnostic information about it. However, studies for structural diagnostics are mainly concentrated in the field of chemistry and medicine [13].

In this paper, we suggest aggregating the data obtained from the measuring devices at a digital substation with structural information about the substation. This approach is based on a change in the correlation dependence between the measured parameters, which makes it possible to assess the structural integrity of the system being diagnosed. In addition, a dynamic estimation of the correlation dependence will allow to predict the change in the correlation dependence, which allows for predictive diagnostics.

One of the main tasks for implementing the proposed approach is the transformation of heterogeneous sources of information about the state of the system to a single view. Based on the method of diakoptics, it is proposed to convert to the form of electrical circuits. Let's consider the scheme on a concrete example - a digital substation. To simulate the substation, we use its simplified structure, shown in Fig. 1 [14]. This scheme include next blocks: HV, LV - high and low voltage; CB1 and CB2 - circuit breakers 1 and 2; CT1 and CT2 - measurement current transformers 1 and 2; VT1 and VT2 - measurement voltage transformers 1 and 2; PT - power transformer.

Stage 1 of the substation scheme, shown in the figure 1, includes a current transformer, a voltage transformer and a circuit breaker on the high voltage side. In this case, it is worth noting that part III of the scheme is similar to part I.

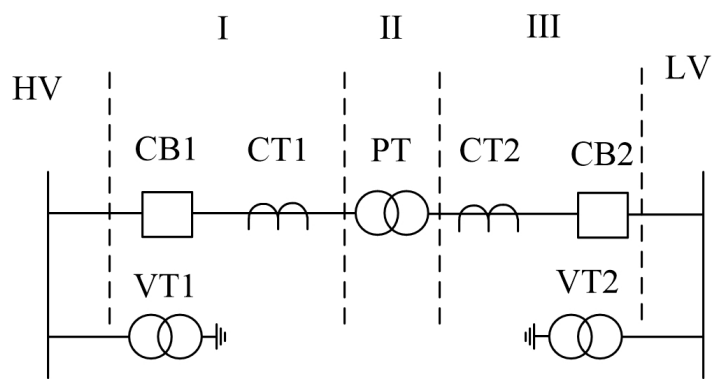

Fig. 1. The substation scheme.

The imitation model of part I of the substation scheme, that was created on the basis of operational amplifiers (OA), is represented in Fig. 2. This imitation model includes next blocks: R2, R8 - feedback impedances equivalent to the detected voltage and current transformer parameter; R1, R7 - compensation resistors to provide a gain factor $\mathrm{Kg}=1$; blocks 1 and 5 are responsible for diagnosing the voltage and current transformer; blocks 2 and 6 are responsible for the inversion of the signal; blocks 3 and 7 designate the element of comparison or tripping of relay protection (RP); block 4 is responsible for the operation of the circuit breaker and the disconnection of the HV. Part I functions as follows: from the $\mathrm{CT}$ and $\mathrm{CV}$, measurement information is obtained, blocks 1, 5 mimic the disturbances in the operation of the $\mathrm{CV}$ and $\mathrm{CT}$, respectively - when the $\mathrm{CT}$ and $\mathrm{CV}$ deviate, the resistances R2 or R8 change, and the measured current or voltage values change. For the convenience of calculating the gain factors OA1 and OA5, an inverting amplifier is taken as the basis of blocks 1 and 5 . 


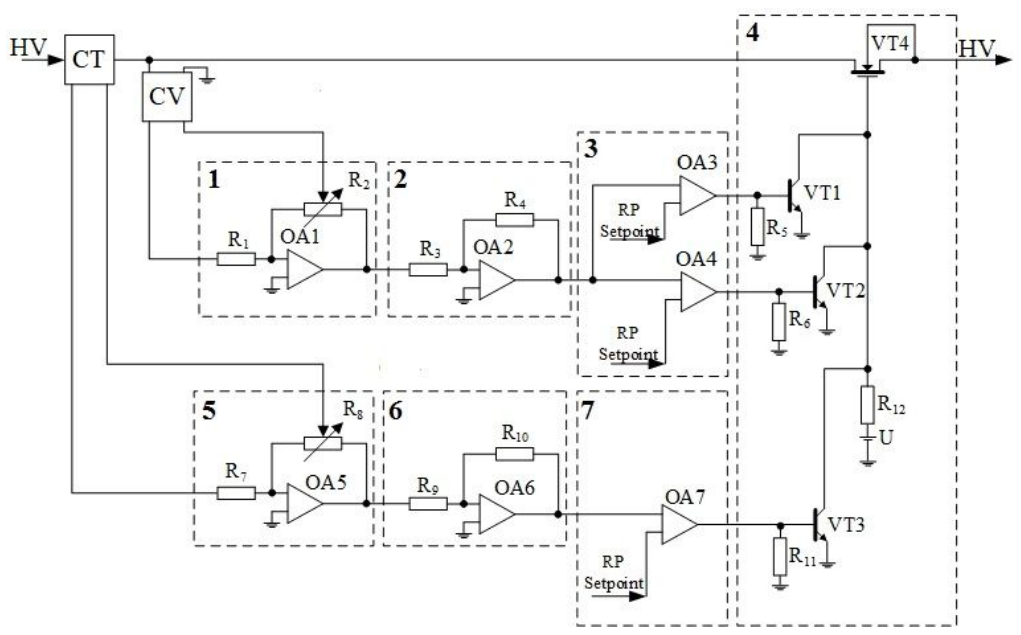

Fig. 2. The part I of the substation scheme.

Further, in order to obtain a positive signal, the units 2 and 6 convert the negative signal into a positive one, and the signals from the current and voltage transformers (with them going the same $50 \mathrm{~Hz}$ alternating voltage) are rectified, that is, they become constant. After that, in block 3, the measured voltage value is compared with the pick-up value of the RP (upper and lower limits). Block 7 compares the measured current value with the pick-up value of the RP (upper limit). Block 4, based on the information from blocks 3 and 7, disables the high-voltage line in the event that the values of the monitored parameters go beyond allowable limits.

The imitation model of part II of the substation scheme is shown in Fig. 3. This imitation model includes next blocks: CT - current transformer, built-in power transformer; TS - temperature sensor of power transformer. For example, a simplified case is considered in which the acquisition of information from a power transformer is carried out from two sources of CT and TS.

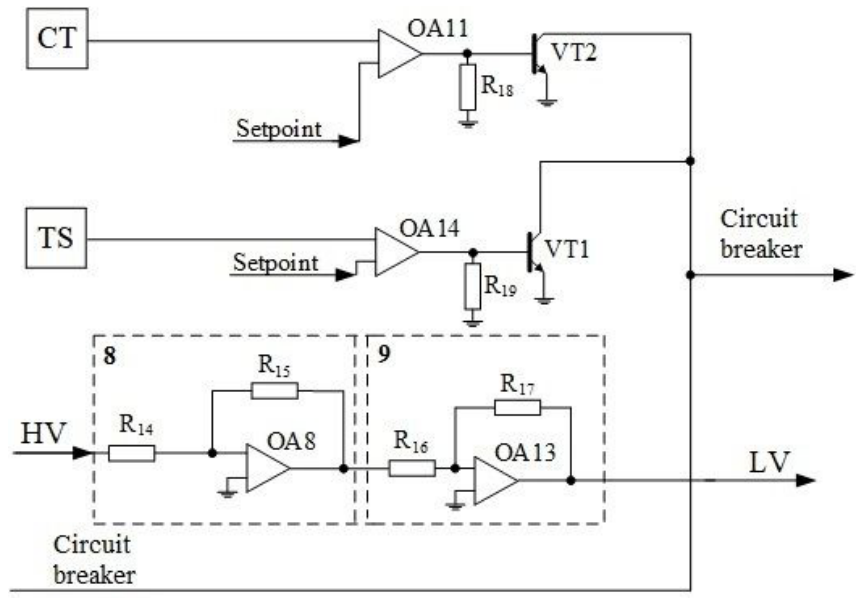

Fig. 3. The part II of the substation scheme.

The workflow of the scheme of Part II (Fig. 3) consists in obtaining information from sources and comparing them with the limiting values. If the monitored value exceeds the permissible limit, a signal is sent to the circuit breaker and the power transformer is 
switched off. Block 8 simulates the operation of a power transformer based on the use of $K g$. Block 9 converts the negative signal received from block 8 to positive for the further operation of the circuit.

\section{Results and discussion}

To test the efficiency of the proposed method, the circuit was modeled on three operational amplifiers [15]. The simulation was carried out in the MicroCap environment. For example, the following parameters are specified in the schema:

1) As the input signal, a voltage equal to $\mathrm{Uin}=75 \mathrm{mV}$ is taken;

2) As the output voltage, Uout $=5 \mathrm{~V}$.

The gain with negative feedback is determined by their ratio and is equal to $\mathrm{Kg} \approx 66.7$. When $\mathrm{R} 1=\mathrm{R} 3, \mathrm{R} 5=\mathrm{R} 4, \mathrm{R} 6=\mathrm{R} 7: K_{g}=\left(\frac{2 \cdot R 1}{R 2}+1\right) \cdot \frac{R 6}{R 5}$. In case $\frac{2 \cdot R 1}{R 2}=6,67$ and $\frac{R 5}{R 6}=10 \quad$ and $\quad R 2=R 4=R 5=10 \quad \mathrm{kOhm}: \quad R 6=10 \cdot R 5, \quad R 7=R 6=10 \cdot 10 \cdot 10^{3}=100$ $\mathrm{kOhm}, R 1=R 3=28,3 \mathrm{kOhm}$. The output voltage is: Uout $=\mathrm{Kg} \cdot \mathrm{Uin}$. The results of simulation the output signal (out point) are shown in Fig. 4.
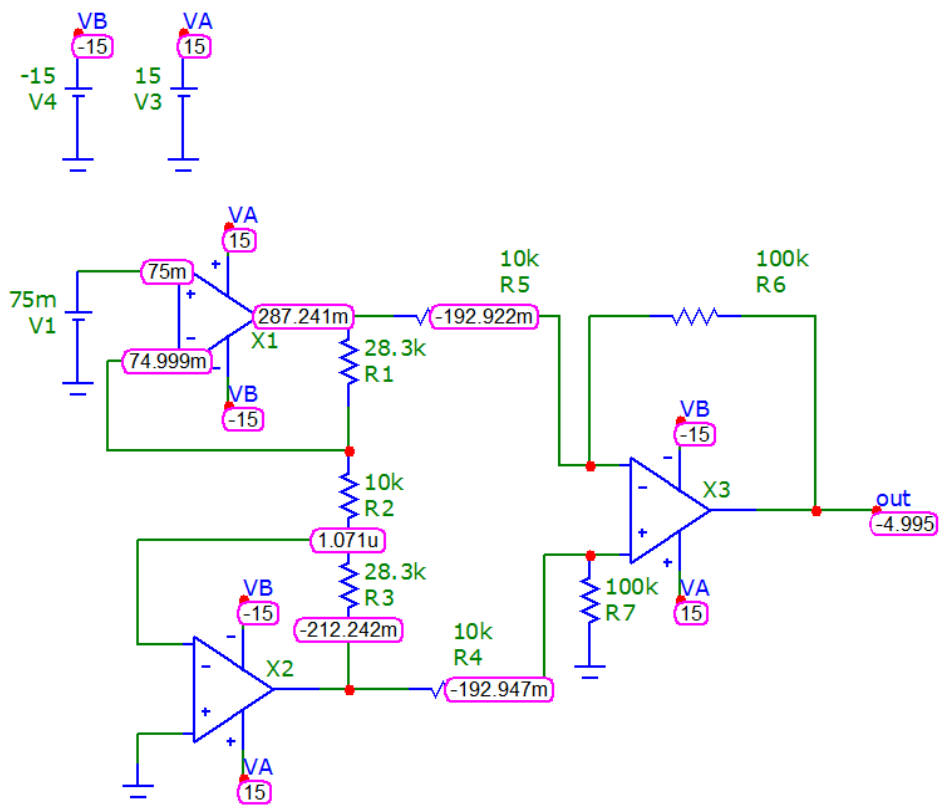

Fig. 4. Calculation results under baseline conditions.

To study the correlation between the resistance of circuit elements and the output signal, the resistance of R1 and R6 is modeled to eliminate compensation in calculating the output signal. To determine the degree of influence of each of these resistances, the theory of experimental planning is used $[16,17]$, respectively, R1 and R6 act as factors, and the output signal as a response. The initial value of the factors is the center of the plan. As variation intervals for R1, 0,5 kOhm is taken, for R6 $5 \mathrm{kOhm}$ is taken. To simplify the analysis, the number of levels of each factor is 2 . The planning matrix for two factors in this example is as follows (Table 1). 
Table 1. Planning Matrix $2^{2}$

\begin{tabular}{|c|c|c|c|c|c|}
\hline $\begin{array}{c}\text { Experiment } \\
\text { nomber }\end{array}$ & $x_{1}$ & $\begin{array}{c}R l, \\
\mathrm{kOhm}\end{array}$ & $x_{2}$ & $\begin{array}{c}R 6, \\
\mathrm{kOhm}\end{array}$ & $U_{\text {out }}, \mathrm{V}$ \\
\hline 1 & -1 & 20 & -1 & 95 & 4,719 \\
\hline 2 & 1 & 12 & -1 & 95 & 4,790 \\
\hline 3 & -1 & 8 & 1 & 105 & 5,195 \\
\hline 4 & 1 & 5 & 1 & 105 & 5,274 \\
\hline
\end{tabular}

The correlation coefficient is determined by the formula [18]:

$$
\rho_{x, y}=\frac{\operatorname{Cov}(X . Y)}{\sigma_{x} \cdot \sigma_{y}}
$$

where $\operatorname{Cov}(X . Y)=\frac{1}{n} \sum_{i-1}^{n}\left(x_{i}-\bar{x}_{i}\right)\left(y_{i}-\bar{y}_{i}\right), \sigma_{\mathrm{x}}$ and $\sigma_{\mathrm{y}}-$ is the standard deviations in the matched rows. On the basis of data in Table 1, the following values of the correlation coefficients are given in Table 2.

Table 2. The values of the correlation coefficient

\begin{tabular}{|c|c|c|c|}
\hline & $R 1$ & $R 6$ & $U_{\text {out }}$ \\
\hline$R 1$ & 1 & & \\
\hline$R 6$ & 0 & 1 & \\
\hline$U_{\text {out }}$ & 0,154 & 0,988 & 1 \\
\hline
\end{tabular}

Further, to evaluate the developing defects in the circuit element, the correlation coefficient was calculated with a decrease in the center of the plan (center of the interval) $\mathrm{R} 1$ to $0.5 \mathrm{kOhm}$ with a variation interval of $0.5 \mathrm{kOhm}$ and with a constant center of the R6 interval, resulting in the following results (Table 3).

Table 3. The values of the correlation coefficient when the center of the plan is changed

\begin{tabular}{|c|c|c|}
\hline $\begin{array}{c}\text { Center of the } \\
\text { plan } R 1, \mathrm{kOhm}\end{array}$ & $\begin{array}{c}\text { Correlation coefficient } \\
R 1 \text { and } U_{\text {out }}\end{array}$ & $\begin{array}{c}\text { Correlation coefficient } \\
R 6 \text { and } U_{\text {out }}\end{array}$ \\
\hline 28,3 & 0,154 & 0,988 \\
\hline 25,0 & 0,163 & 0,987 \\
\hline 20,0 & 0,176 & 0,984 \\
\hline 10,0 & 0,214 & 0,977 \\
\hline 0,5 & 0,268 & 0,963 \\
\hline
\end{tabular}

From Table 3 it can be seen that when the interval center is altered, which simulates the developing defect, the correlation coefficient changes, due to which it is possible to determine the degree of deviation from the norm in the work of the element under consideration.

With an increase in the range of variation of $\mathrm{R} 1 \mathrm{up}$ to $1 \mathrm{kOhm}$ the correlation coefficient varies from 0.298 to 0.479 , which allows a more detailed assessment of the degree of disturbance. With a variation range of $\mathrm{R} 1$ equal to $1.5 \mathrm{kOhm}$, the correlation coefficient varies from 0.423 to 0.627 . With a further increase in the variation interval $\mathrm{R} 1$, for example, up to $5 \mathrm{kOhm}$, the correlation coefficient changes from 0.816 to 0.925 , while the correlation coefficient between R6 and Uout varies from 0.577 to 0.377 .

Based on the conducted studies, it follows that as the range of variation of $\mathrm{R} 1$ increases, the correlation coefficient increases, which is obvious, since the step is too large and such a 
change in resistance exerts a larger influence on Uout than in R6. However, the detailing of the degree of damage is reduced, as in the case of too small a range of variation. In this particular case, the most optimal option is the range of variation equal to $3.5-5 \%$ of the center of the plan.

To determine the structural integrity of the circuit under study, the correlation coefficient was studied by breaking one of the branches of the circuit. To investigate the effect of such a violation, the value of the resistance R1 was chosen to be equal to infinity and zero at a variation interval of R6 equal to $5 \mathrm{kOhm}$. The results of calculating the correlation coefficients are given in Table 4.

Table 4. The calculations of correlation coefficient values for $\mathrm{R} 1=0$ and $\mathrm{R} 1=\infty$.

\begin{tabular}{|c|c|c|c|}
\hline & $R 1$ & $R 6$ & $U_{\text {out }}$ \\
\hline$R 1$ & 1 & & \\
\hline$R 6$ & 0 & 1 & \\
\hline$U_{\text {out }}$ & 0,999 & 0,011 & 1 \\
\hline
\end{tabular}

According to Table 4, the correlation coefficient of $\mathrm{R} 1$ to the $\mathrm{U}_{\text {out }}$ take a value very close to unit, this allows us to identify the breaking of bonds based on the correlation.

\section{Conclusion}

The conducted researches show that diagnostics of complex technical systems should be based on the analysis of all measurement data in a complex. To solve this problem, an effective way is to create a simulation model by converting the heterogeneous components of the system to a single view. Based on the methods of diakoptics, a simulation model of a digital substation has been developed. A diagnostic method is proposed, in which the change in the correlation coefficient is used as the main diagnostic feature. It is obtained that by controlling the change in the correlation coefficient, it is possible to estimate the developing defect of the element being diagnosed, and under the condition of a sharp jump in the correlation coefficient - about structural disturbance in the system being diagnosed. As a result of the conducted researches, the operability of structural and parametric diagnostics on the basis of correlation analysis was proved.

The reported study was funded by RFBR according to the research project № 18-38-00950. This article is based on results obtaned by using equipment of shared facility "Diagnosis and energyefficient electrical equipment" (NPI).

\section{References}

1. S.N. Rykovanov, V.I. Ukhov, S.R. Slabospitssky, I.O. Kovtsova, J. Releyshchik, 1, 32 (2014)

2. T.V. Drozdova, N.E. Elov, A.P. Morozov, J. Energy of a Uniform Network, 3, 54 (2016)

3. In Skolkovo opened digital substation $110 \mathrm{kV}$ Medvedevsky. Available at: http://www.en-prom.ru/2018/06/05/v-skolkovo-otkry-ta-tsifrovaya-ps-110-kvmedvedevskaya/

4. L.A. Daryan, A.G. Mordkovich, G.M. Tsfasman, Electro. Electrical engineering, electric power industry, electrotechnical industry, 5, 22 (2010) 
5. V.I. Dubrov, R.G. Oganyan, D.V. Shaykhutdinov, E.V. Kirievsky, T.N. Kruglova, N.D. Narakidze, Fundamental Research, 9, 16 (2016)

6. V.I. Dubrov, R.G. Oganyan, N.D. Narakidze, G.K. Aleksanyan, J.Eng.Aplliend Sci., 12(2), 276 (2017)

7. A. Vanin, S. Aleshin, R. Nasirov, D. Novikov, V. Tulsky, Information, 7, 42, (2016)

8. D.K. Eltyshev, V.V. Boyarshinova, Proceedings of the 19th International Conference on Soft Computing and Measurements, SCM, 157 (2016)

9. J. Pathak, Y. Jiang, V. Honavar, J. McCalley, Proceedings of the Annual Hawaii International Conference on System Sciences, 10, 241a (2006)

10. Y. Ma, Z. Guo, J. Su, Y. Chen, X. Du, Y. Yang, C. Li, Y. Lin, Y. Geng, POWERCON 2014 - 2014 International Conference on Power System Technology: Towards Green, Efficient and Smart Power System, Proceedings, 740 (2014)

11. W. Sun, M. Shi, C. Zhang, J. Zhao, X. Song, Automation in Construction, 92, 23 (2018)

12. J. Zhang, M. Li, L. Zhang, J. Yang, Proceedings - 5th International Conference on Internet Computing for Science and Engineering, ICICSE 2010, 188 (2011)

13. V. Metsis, H. Huang, O.C. Andronesi, F. Makedon, A. Tzika, Oncology Reports 28(4) 1413 (2012)

14. P. Horovits, U. Hill, The art of circuitly (MIR, Moscow, 1993)

15. R.G. Oganan, G.I. Saenko, Information and measuring systems and technologies: Proceedings of scientific articles on the materials of Intern. scientific-technical conference, 23 (2016)

16. N.I. Gorbatenko, M.V. Lankin, D.V. Shaykhutdinov, Experiment planing (SRSPU, Novocherkassk, 2007)

17. M.A. Kharchenko, Correlation analysis (VGU, Voronegh, 2008)

18. L.D. Rogkova, V.S. Kozulin, Electrical equipment of power stations and substations (EnergyAtomIzdat, Moscow, 1987) 